\title{
Sensitivity of the Packet Level Performance Metrics to the Primary Channel Holding Time Distribution in Cognitive Radio Networks with VoIP-traffic
}

\author{
S. Lirio Castellanos-Lopez, \\ Felipe A. Cruz-Pérez ${ }^{1}$ \\ ${ }^{1}$ Electrical Engineering Department \\ CINVESTAV-IPN \\ Mexico City, Mexico \\ $\{\underline{\text { lirio, facruz }\} @ \text { cinvestav.mx }}$
}

\author{
Genaro Hernandez-Valdez ${ }^{2}$ \\ ${ }^{2}$ Electronics Department \\ UAM-A \\ Mexico City, Mexico \\ ghv@correo.azc.uam.mx
}

\author{
Mario E. Rivero-Angeles ${ }^{3}$ \\ ${ }^{3}$ Communication Networ ks \\ Laboratory \\ CIC-IPN \\ Mexico City, Mexico \\ mriveroa@ipn.mx
}

\begin{abstract}
In this paper, the performance of VoIP trafficbased cognitive radio networks (CRNs) with different primary channel holding time (CHT) distribution (specifically, negative exponential, log-normal, and Coxian) is investigated. From the mathematical point of view, a joint connection level and packet level analysis considering the primary CHT to be Coxian distributed is developed. The developed mathematical analysis allows evaluating system performance at both connection and packet levels. Then, system performance is evaluated in terms of packet dropping probability and mean packet delay considering a target VoIP session blocking probability of cognitive users. Numerical results for CHT log-normally and Coxian distributed are shown for different values of their first three standardized moments. Numerical results reveal that packet level performance metrics are practically insensitive to the probability distribution of the primary CHT. Thus, the analytical performance evaluation can be performed considering the negative exponential distribution for the sake of simplicity and mathematical tractability.
\end{abstract}

Keywords: Cognitive radio networks, VoIP traffic, primary channel holding time probability distribution, session blocking probability, packet dropping probability, mean packet delay.

\section{INTRODUCTION}

Cognitive radio (CR) technology enables secondary users (SUs) of a dynamic spectrum access network to utilize the spectrum more efficiently in an opportunistic fashion without interfering with primary users (PUs) (licensed holders of the spectrum). With the development of CR technology, it is believed that the introduction of voice over IP (VoIP) services in CR networks (CRNs) is significant and useful. However, there is not very much research on the performance of CRNs with VoIP traffic [1]-[5]. Developing analytical tools for the performance evaluation of CRNs under VoIP traffic that effectively capture relevant aspects at both call and packet level of these complex networks represents a very important research task. Analysis of CRNs have been commonly performed under the unrealistic assumption that channel holding time (CHT) for PUs is exponentially distributed [6], [7]. Although, the exponential model is simple and mathematically tractable, field measurements have shown that CHT is not exponentially distributed in real world applications [8], [26]. Hence, some research is necessary to both asses the applicability range of simpler models and develop more versatile and realistic models. In this research direction, CHT distribution has been extensively studied during the last two decades [9]-[14], [26], [27]. Furthermore, almost all of the references cited above reported the CHT distribution to be different to the exponential one (such as log-normal, Gamma, generalized Pareto and Weibull type). However, the application of these non-exponential models will lead to the loss of the Markov property required in the queuing analysis. To overcome this drawback, authors in [15] employ a twophase Coxian distribution for the CHT in the primary network (PN) and derive the distribution for the duration of the channel idle time (i.e., spectrum white spaces). In [15], the distribution of the spectrum white spaces when the CHT is log-normally distributed is computed by means of computer simulation, and the results are compared with those obtained analytically.

On the other hand, previous works dealing with CRNs with VoIP traffic have not considered call blocking probability as performance metric and only [4] considers packet delay as performance metric. The authors of [1]-[5] developed their performance analysis for a fixed total number of VoIP users. Then, with the analytical models developed in [1]-[3], [5], it is possible neither to capture the dynamic of arrival and departure of VoIP sessions nor to evaluate new call blocking probability. Only packet level (i.e., average throughput, packet dropping probability, and queue length) performance metrics are evaluated in [1]-[3], [5]. In a related work [4], the effective bandwidth and effective capacity concepts are used to analyze, respectively, the source traffic flow and the channel service process in CRNs with VoIP traffic. In [16] and [19], we developed a joint call and packet level teletraffic model for the performance evaluation of one proposed local call admission control (CAC) strategy for VoIP traffic over CRNs. However, the analysis developed in [16] and [19] considers that the primary CHT is exponentially distributed. In [24] the use of Coxian distribution of arbitrary order to model CHT in the PN is considered. However, the study carried out in [24] focuses only on the performance at the connection level while performance at the packet level is ignored.

In this work, the performance of CRNs from a VoIP traffic perspective with different primary CHT probability distribution is studied. Our study focuses on both connection level and packet levels by evaluating the packet dropping probability and the mean packet delay of the secondary users 
(SUs). From the mathematical point of view, a joint connection and packet level analysis considering the primary CHT to be Coxian distributed is developed. The importance of adopting the Coxian model instead of the exponential one is due to the fact that it can be used to match any real environment CHT distribution arbitrarily closely [25], while retaining the underlying Markovian properties of the distribution. Our developed mathematical framework considers the use of adaptive modulation and coding (AMC), packet buffering, proportional fair sharing scheduling, and local CAC mechanism. The developed teletraffic model captures the arrivals and completions of VoIP sessions, on/off activity detection, and periodic and constant length packet generation (of active sessions) of individual VoIP sessions. To achieve mathematical tractability, the analysis employs the time-scale decomposition technique [17]-[19]. Building on this, the novelty of our contribution is to investigate the impact of the primary CHT probability distribution on the packet level performance metrics of VoIP traffic-based CRNs with AMC and buffering.

The rest of this paper is structured as follows. System model and general assumptions are given in Section II. The mathematical analysis for performance evaluation of VoIPtraffic based CRNs with AMC and Coxian distributed primary CHT is developed in Section III. Numerical results are analyzed in Section IV, before concluding remarks are exposed in Section V.

\section{SYSTEM MODEL}

In the case of the CRN studied in this work, VoIP users are SUs and they occupy wireless channels transparently, while PUs have absolute priority over SUs. It means that when VoIP packet is in processing and a PU arrives, it seizes the channel. Hence, the secondary call is interrupted, and its packets are queued into the buffer (the buffer length is denoted by $Q$ ). Once there are no more primary packets in the CRN, those unoccupied channels continue to dispose packets of SUs. System performance at the downlink of a wireless CRN is addressed, i.e., only the local domain at the receiving end is considered. We assume a single base station (i.e. evolved node $\mathrm{B}$ or eNB in LTE vocabulary) that performs data transmission over $M$ shared channels. It is assumed that users requesting a VoIP session are able to connect (and stay connected up to the end of their sessions) to this base station, that is, no handoff mechanism is considered (typical in low mobility scenarios). AMC scheme is considered, to this end, the cell is divided into $z$ coverage zones. A SU located in the $i$-th coverage is allowed to use the $i$-th data rate, denoted by $R_{b}{ }^{(i)}$.

The arrival of both primary and secondary users is assumed to be independent Poisson processes with arrival rate $\lambda^{(P)}$ and $\lambda^{(S)}$, respectively. It is important to notice that in this work, the average time between arrivals is considered to be in the order of seconds while the frame duration (inter-arrival packet time) is in the order of a few milliseconds. As such, it is possible to use the time-scale decomposition technique to analyze the CR system under VoIP traffic. As it is shown in Section III, connection level analysis is performed in a continuous time basis while the packet level analysis is performed in a discrete time basis. The effect of packet level on the connection level is captured by connecting the resulting packet and connection level queuing systems. On the other hand, call duration is also considered to be exponentially distributed with mean $1 / \mu^{(S)}$ for SUs while for PUs call duration is considered arbitrary order Coxian distributed with finite mean $1 / \mu^{(P)}$. Statistical characterization of telephone conversations conducted in the 1960s at Bell Laboratories [20] resulted in the main finding that a telephone conversation can be represented by the ON (talk spurt)-OFF (silence) pattern. Furthermore, it was found that the duration of ON and OFF periods can approximately be modeled by an exponential distribution with mean value of 1.2 and $1.8 \mathrm{~s}$, respectively. In this work, without loss of generality, we assume that these periods are geometrically distributed with parameters $P_{O N-O F F}$ and $P_{O F F-O N}$, respectively. The parameter $P_{\text {ON-OFF }}\left(P_{\text {OFF-ON }}\right)$ represents the probability that a call that is currently in the active (silent) period turns to a silent (active) period. During the active periods, we assume that the voice samples are generated with a constant bit rate. In the VoIP call model used in this work, each voice packet is composed of protocol headers (RTP, UDP and IP) and the payload. It is considered that all users use the same voice codec, and hence both the size of VoIP packet and packet interval are identical. In our model, we consider that during the active periods the voice packets are periodically generated every $T$ seconds. A typical value used for $T$ is $20 \mathrm{~ms}$ [21]. Also, packet spacing of 10 and $20 \mathrm{~ms}$ has been used in [22].

We also consider a CAC strategy based on the total number of users (both active and inactive sessions) in the system. In this strategy, when there are $S$ users in the system and an arrival occurs, the arriving user is blocked. A similar CAC based on the theoretical capacity estimation was considered in [23]. It is important to notice that this strategy is analyzed at both connection and packet level due mainly to the complexity of the proposed CR with AMR system model as explained in more detail in the following section.

\section{JOINT CALL-LEVEL AND PACKET LEVEL ANALYSIS}

In this section, considering that the primary CHT is Coxian distributed, the mathematical methodology proposed in [19] is employed to develop a joint connection-level and packet-level analytical model for the performance evaluation of a VoIP traffic-Based CRN with AMC and buffering. Building on this, mathematical expressions for the blocking and packet dropping probabilities are obtained. As in [19], the analysis is performed at two levels employing the time-scale decomposition technique: connection level and packet level. At the packet level, the total number of users in the system (both active and inactive) is considered to be fixed. As such, the probability of having a certain amount of active users and packets to be transmitted can be calculated. Both analyses are necessary in order to find the performance parameters for a dynamic system where users continually enter and leave the system as well as VoIP sessions are continually switching between active or inactive states. Note that for the CAC strategy considered (based on $S$ ), only the connection level is necessary in order to determine if a new call is accepted or rejected. This is because the system only has to check the number of total users. 


\section{A. Connection Level Analysis}

In this section, a teletraffic analysis for the performance evaluation of VoIP traffic-based CRNs with arbitrary order Coxian distributed CHT in the primary network and AMC is developed. The developed teletraffic model considers that the admission parameter $S$ is a real number. That is, a fractional threshold on $S$ is considered, i.e., with probability $p_{\text {ses }}=S-\lfloor S\rfloor$, one secondary session is accepted when there are $\lfloor S\rfloor$ users (secondary and primary) in the system and with probability $\left(1-p_{\text {ses }}\right)$ the new secondary session is blocked. Due to the fact, that at the connection level, the total number of users varies according to Poisson arrival processes and exponentially distributed session times, a continuous time analysis is suitable. Thus, the Markov chain described in Fig. 1 is proposed in order to perform the connection level analysis. The number of state variables required for modeling this system through a multidimensional birth and death process is $w+z$ ( $w$ for each of the phases of the CHT of PUs and $z$ to identify the number of SUs in each quality zone).

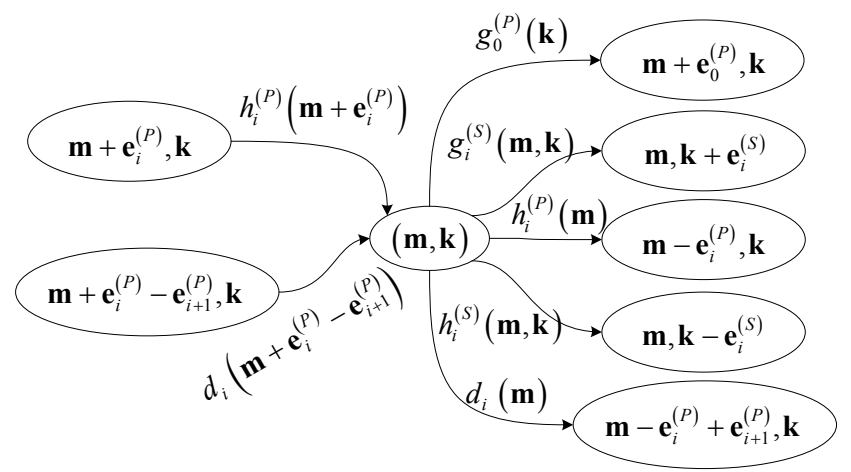

Fig. 1.Transition diagrams for the Markov chain of the CR system with AMC for IP voice traffic for the secondary and primary users at connection level.

Let $m_{i}$ represent the number of PUs in phase $i$-th of its CHT (for $i=1, \ldots, w$ ). Each state variable for SUs is denoted by $k_{j}$ $($ for $j=1,2, \ldots, z)$. Hence, in state $(\mathbf{m}, \mathbf{k})\left(\mathbf{m}=\left[m_{1}, \ldots, m_{w}\right], \mathbf{k}=[\right.$ $\left.\left.k_{1}, \ldots, k_{z}\right]\right)$, there can either be one primary user arrival or a second user arrival at zone $i$ with rate $g_{i}^{(P)}(\mathbf{m}), g_{i}^{(S)}(\mathbf{k})$ respectively. Vector $\mathbf{e}_{i}^{(\boldsymbol{P})}$ is a zero vector of size $w$ with only a 1 in position $i(i=1, \ldots, w)$. Vector $\mathbf{e}_{j}^{(S)}$ is a zero vector of size $w$ with only a 1 in position $j(j=1, \ldots, \mathrm{z})$. On the other hand, in state $(\mathbf{m}, \mathbf{k})$, there can either be one PU's service termination or a SU that leaves the system at zone $i$ with rate $h_{i}^{(P)}(\mathbf{m}), h_{i}^{(S)}($ k) respectively. And there can either be one PU in phase $i$ of its CHT that changes to phase $i+1$ with rate $d_{i}(\mathbf{m})$. Let $\gamma_{i}$ represent the probability that the absorbing state of the arbitrary order Coxian distribution is reached after the $i$-th phase and $\mu_{i}^{(P)}$ represent the inverse of the mean permanence time in the $i$-th phase (for $i=1, \ldots, w$ ). For an $w$-th order Coxian distribution, $\gamma_{w}=1$. Thus, the transition rates shown in Fig. 1 can be expressed as follows

$$
g_{i}^{(P)}(\mathbf{m})=\left\{\begin{array}{l}
\lambda^{(P)} ; \sum_{j=0}^{w-1} m_{j} \geq 0 ; \sum_{j=1}^{w} m_{j}<M ; i=1 \\
0 \quad \text { otherwise }
\end{array}\right.
$$

$$
g_{i}^{(S)}(\mathbf{m}, \mathbf{k})=\left\{\begin{array}{l}
p_{i} \lambda^{(S)} ; \quad k_{i} \geq 0 ; \quad \sum_{j=1}^{w} m_{j}+\sum_{j=1}^{z} k_{j}<\lfloor S\rfloor ; i=1,2 \\
p_{\text {ses }} p_{i} \lambda^{(S)} ; \quad k_{i} \geq 0 ; \sum_{j=1}^{w} m_{j}+\sum_{j=1}^{2} k_{j}=\lfloor S\rfloor ; i=1,2 \\
0 \quad \text { otherwise }
\end{array}\right.
$$

$$
h_{i}^{(P)}(\mathbf{m})=\left\{\begin{array}{l}
\gamma_{i} m_{i} \mu_{i}^{(P)} ; \sum_{j=1}^{w} m_{j} \leq M ; i=1, \ldots, w \\
0 \quad \text { otherwise }
\end{array}\right.
$$

$$
\begin{aligned}
& h_{i}^{(S)}(\mathbf{m}, \mathbf{k})=\left\{\begin{array}{l}
k_{i} \mu^{(S)} ; \sum_{j=1}^{2} k_{j} \leq\lceil S\rceil ; i=1,2 \\
0 \quad \text { otherwise }
\end{array}\right. \\
& d_{i}(\mathbf{m})= \begin{cases}\left(1-\gamma_{i}\right) m_{i} \mu_{i}^{(P)} \quad ; \mathrm{m}_{i+1} \geq 0 ; m_{i} \leq M \\
0 & ; \text { otherwise }\end{cases}
\end{aligned}
$$

The valid states space is given by

$$
\Omega_{\text {ses }}=\left\{\mathbf{m}, \mathbf{k} \mid \begin{array}{l}
\mathbf{m}=\left[m_{1}, \ldots, m_{w}\right] ; 0 \leq m_{i} \leq M ; 0 \leq \sum_{i=1}^{w} m_{i} \leq M, i=1, \ldots, w \\
\mathbf{k}=\left[k_{1}, \ldots, k_{z}\right] ; 0 \leq \sum_{i=1}^{z} k_{i} \leq\lceil S\rceil, i=1, \ldots, z
\end{array}\right\}
$$

As such, considering that $\mathrm{P}(\mathbf{m}, \mathbf{k})$ represents the steady state probability that the system is in state $(\mathbf{m}, \mathbf{k})$, the system in statistical equilibrium can be described according to the following equation:

$$
\begin{aligned}
& {\left[\begin{array}{l}
g_{0}^{(P)}(\mathbf{m})+\sum_{i=1}^{z} g_{i}^{(S)}(\mathbf{m}, \mathbf{k})+\sum_{i=1}^{w} h_{i}^{(P)}(\mathbf{m}) \\
+\sum_{i=0}^{z} h_{i}^{(S)}(\mathbf{m}, \mathbf{k})+\sum_{i=1}^{w} d_{i}(\mathbf{m})
\end{array}\right] P(\mathbf{m}, \mathbf{k})=} \\
& g_{0}^{(P)}\left(\mathbf{m}-\mathbf{e}_{0}^{(P)}\right)+\sum_{i=1}^{z} g_{i}^{(S)}\left(\mathbf{m}, \mathbf{k}-\mathbf{e}_{i}^{(S)}\right) P\left(\mathbf{m}, \mathbf{k}-\mathbf{e}_{i}^{(S)}\right) \\
& +\sum_{i=1}^{w} h_{i}^{(P)}\left(\mathbf{m}+\mathbf{e}_{i}^{(P)}\right) P\left(\mathbf{m}+\mathbf{e}_{i}^{(P)}\right)+\sum_{i=1}^{z} h_{i}^{(S)}\left(\mathbf{m}, \mathbf{k}+\mathbf{e}_{i}^{(S)}\right) P\left(\mathbf{m}, \mathbf{k}+\mathbf{e}_{i}^{(S)}\right) \\
& +\sum_{i=1}^{w} d_{i}\left(\mathbf{m}+\mathbf{e}_{i}^{(P)}-\mathbf{e}_{i+1}^{(P)}\right) P\left(\mathbf{m}+\mathbf{e}_{i}^{(P)}-\mathbf{e}_{i+1}^{(P)}\right)
\end{aligned}
$$

The blocking probability for secondary calls is given by:

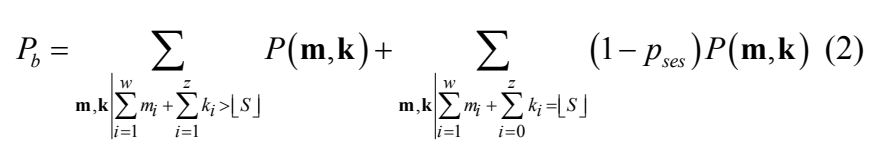

\section{A. Packet Level Analysis}

Since at the packet level, events occur only at the beginning of the time slots, a discrete time analysis is preferred. Thus, the mathematical analysis developed in this section assumes that the system evolves in a discrete time basis where the unit of time is considered to be the frame time duration, $T$. Since the value of the frame time duration is sufficiently small compared to the inter-arrival time of users to the system, the service time in the system, and the active/inactive periods, it is assumed that 
Table I. Transitions From a Generic State $\mathbf{n}=\left[n_{1}, n_{2}, n_{3}\right]$ of $W=\left(n_{1}, n_{2}, n_{3}\right)$

\begin{tabular}{|c|c|c|c|}
\hline Activity & Destination state & Rate & Conditions $^{*}$ \\
\hline There are no changes in the number of active SUs. & $\left(n_{1}, n_{2}, n_{3}+x-L_{p}^{\prime}\right)$ & $\begin{array}{l}\left(1-P_{O N \rightarrow O F F}^{1}\right)^{n_{1}}\left(1-P_{O N \rightarrow O F F}^{2}\right)^{n_{2}} \\
\left(1-P_{O F F \rightarrow O N}^{1}\right)^{k_{1}-n_{1}}\left(1-P_{O F F \rightarrow O N}^{2}\right)^{k_{2}-n_{2}}\end{array}$ & - \\
\hline A SU in zone 1 becomes active. & $\left(n_{1}+1, n_{2}, n_{3}+x-L_{p}^{\prime}\right)$ & $\left(1-P_{O N \rightarrow O F F}^{1}\right)^{n_{1}} \beta_{1}\left(1-P_{O N \rightarrow O F F}^{2}\right)^{n_{2}}\left(1-P_{O F F \rightarrow O N}^{2}\right)^{k_{2}-n_{2}}$ & $n_{1}<k_{1}$ \\
\hline A SU in zone 1 goes to the OFF mode. & $\left(n_{1}-1, n_{2}, n_{3}+x-L_{p}^{\prime}\right)$ & $\alpha_{1}\left(1-P_{O F F \rightarrow O N}^{1}\right)^{k_{1}-n_{1}}\left(1-P_{O N \rightarrow O F F}^{2}\right)^{n_{2}}\left(1-P_{O F F \rightarrow O N}^{2}\right)^{k_{2}-n_{2}}$ & $n_{1}>0$ \\
\hline There is one more active SU in zone 2 . & $\left(n_{1}, n_{2}+1, n_{3}+x-L_{p}^{\prime}\right)$ & $\left(1-P_{O N \rightarrow O F F}^{1}\right)^{n_{1}}\left(1-P_{O F F \rightarrow O N}^{1}\right)^{k_{1}-n_{1}}\left(1-P_{O N \rightarrow O F F}^{2}\right)^{n_{2}} \beta_{2}$ & $n_{2}<k_{2}$ \\
\hline An active SU in zone 2 goes to the OFF mode. & $\left(n_{1}, n_{2}-1, n_{3}+x-L_{p}^{\prime}\right)$ & $\left(1-P_{O N \rightarrow O F F}^{1}\right)^{n_{1}}\left(1-P_{O F F \rightarrow O N}^{1}\right)^{k_{1}-n_{1}} \alpha_{2}\left(1-P_{O F F \rightarrow O N}^{2}\right)^{k_{2}-n_{2}}$ & $n_{2}>0$ \\
\hline There is one new active SU in both zones. & $\left(n_{1}+1, n_{2}+1, n_{3}+x-L_{p}^{\prime}\right)$ & $\left(1-P_{O N \rightarrow O F F}^{1}\right)^{n_{1}} \beta_{1}\left(1-P_{O N \rightarrow O F F}^{2}\right)^{n_{2}} \beta_{2}$ & $n_{1}<k_{1} ; n_{2}<k_{2}$ \\
\hline $\begin{array}{l}\text { One SU goes to the OFF mode in zone } 1 \text { and one } \\
\text { SU becomes active in zone } 2 .\end{array}$ & $\left(n_{1}-1, n_{2}+1, n_{3}+x-L_{p}^{\prime}\right)$ & $\alpha_{1}\left(1-P_{O F F \rightarrow O N}^{1}\right)^{k_{1}-n_{1}}\left(1-P_{O N \rightarrow O F F}^{2}\right)^{n_{2}} \beta_{2}$ & $n_{1}>0 ; n_{2}<k_{2}$ \\
\hline $\begin{array}{l}\text { A SU in zone } 1 \text { goes to the ON mode while a SU } \\
\text { in zone } 2 \text { goes to the OFF mode. }\end{array}$ & $\left(n_{1}+1, n_{2}-1, n_{3}+x-L_{p}^{\prime}\right)$ & $\left(1-P_{O N \rightarrow O F F}^{1}\right)^{n_{1}} \beta_{1} \alpha_{2}\left(1-P_{O F F \rightarrow O N}^{2}\right)^{k_{2}-n_{2}}$ & $n_{1}<k_{1} ; n_{2}>0$ \\
\hline $\begin{array}{l}\text { There is one SU that goes to the OFF mode in both } \\
\text { zones. }\end{array}$ & $\left(n_{1}-1, n_{2}-1, n_{3}+x-L_{p}^{\prime}\right)$ & $\alpha_{1}\left(1-P_{O F F \rightarrow O N}^{1}\right)^{k_{1}-n_{1}} \alpha_{2}\left(1-P_{O F F \rightarrow O N}^{2}\right)^{k_{2}-n_{2}}$ & $n_{1}>0 ; n_{2}>0$ \\
\hline
\end{tabular}

*For all the possible transitions the condition $0 \leq n_{3}+x+L_{p}^{\prime} \leq C_{\max }+Q$ must be achieved.

in a frame time duration there can only be one user arrival to the system, or one user ending its session, or one user in OFF mode becomes active, or one in ON mode goes to stand by. The accuracy of this approximation is verified through simulation results. The probability that one SU goes from the ON state to the OFF state, or vice versa, can be calculated, respectively by

$$
\alpha_{i}=\left(\begin{array}{c}
n_{i} \\
1
\end{array}\right) P_{O N \rightarrow O F F}^{i}\left(1-P_{O N \rightarrow O F F}^{i}\right)^{n_{i}-1}
$$

and

$$
\beta_{i}=\left(\begin{array}{c}
k_{i}-n_{i} \\
1
\end{array}\right) P_{O F F \rightarrow O N}^{i}\left(1-P_{O F F \rightarrow O N}^{i}\right)^{k_{i}-n_{i}-1}
$$

where $k_{i}$ is the total number of SUs in zone $i$ and $n_{i}$ is the active sessions of SUs in zone $i$ at the current frame. As such, the number of packets generated by SUs in zone $i$ is simply $n_{i}$ and the total number of packets generated by SUs is $\sum_{i=1}^{z} n_{i}$. Considering the use of the Proportional Fair Sharing scheduling method, the number of packets that can be transmitted in each frame depends on the number of active users in each zone. Hence, the capacity of the system at the current frame can be described as:

$$
C=\left\lfloor\left(\frac{\sum_{i=1}^{z} R_{b}^{(i)} n_{i}}{\sum_{i=1}^{z} n_{i}}\right) T\left(M-\sum_{i=1}^{w} m_{i}\right) / b\right\rfloor
$$

where $b$ is the number of bits per packet and $T$ is the time between consecutive packets of the same user which is also considered as the frame duration. The maximum capacity for the SUs occurs when there are no PUs in the system and all the SUs are in the good zone. This capacity is given by $C_{\max }=\left\lfloor R_{b}^{1} T M / b\right\rfloor$. Note that the system capacity depends also on the number of PUs using resources, $m_{i}$, which account for the CRN. The average bit rate per channel at the current frame can be expressed as: $\bar{R}=\sum_{i=1}^{z} R_{b}^{(i)} n_{i} / \sum_{i=1}^{z} n_{i}$. Hence, the number of transmissions in the frame period is described by $y=\min \left(C, n_{z+1}\right)$, where $C=\left\lfloor\bar{R} T\left(M-\sum_{i=1}^{w} m_{i}\right) / b\right\rfloor$.

Building from this, packets can be lost in a time frame period when the number of packets to transmit is higher than the available resources at the next frame period. Specifically, the number of lost packets in a time slot can be calculated as:

$$
L_{p}^{\prime}=\max \left(0, \sum_{i=1}^{z} n_{i}+n_{z+1}-y-C^{\prime}-Q\right)
$$

where $C^{\prime}$ is the capacity at the next frame period. The capacity $C^{\prime}$ depends on the number of active users in each zone at the next frame period and is given by:

$$
C^{\prime}=\left\lfloor\left(\frac{\sum_{i=1}^{z} R_{b}^{(i)} n_{i}^{\prime}}{\sum_{i=1}^{z} n_{i}^{\prime}}\right) T\left(M-\sum_{i=1}^{w} m_{i}\right) / b\right\rfloor
$$

In order to simplify the analysis, only two zones are considered in the rest of the paper, i.e., a good zone (zone 1 with bit rate $R_{b}^{1}$ bps) and a bad zone (zone 2 with bit rate $R_{b}^{2}$ bps). Indeed, it is considered that zone 1 has a higher bit rate due to the better channel conditions $\left(R_{b}^{1}>R_{b}^{2}\right)$. From this, the number of packets of SUs to be transmitted is given by $n_{3}^{\prime}=n_{3}+x-L_{p}^{\prime}$ where $x=\sum_{i=1}^{2} n_{i}-\min \left(C, n_{3}\right)$ and $n_{3}$ is the number of packets transmitted at the current frame. The aforementioned system can be modeled as a discrete-time Markov chain $W=\left(n_{1}, n_{2}, n_{3}\right)$ with valid states spaces:

$$
\Omega_{p}=\left\{\mathbf{n} \mid 0 \leq n_{i} \leq k_{i}, \quad 0 \leq n_{3} \leq C_{\max }+Q, i=1,2\right\}
$$

where $\mathbf{n}=\left[n_{1}, n_{2}, n_{3}\right]$. As such, a SU is blocked when there are $\lceil S\rceil$ SUs (active or inactive) already in the system. Also, a PU is blocked when PUs are using all the $M$ channels. Note that if SUs occupy one or more of the $M$ channels, a PU can access the system by placing the SU in the OFF mode. Consider $W$ to 
be a discrete time homogeneous Markov chain, Table I summarizes the state transition rates at the packet level. Note that the effect of the PUs is given trough the variable $n_{3}$ which is calculated using the capacity of the system described by (3). This chain is numerically solved using the Gauss-Seidel method by considering the set of lineal equations given by $\boldsymbol{\Pi} \mathbf{P}=\boldsymbol{\Pi}$ where $\Pi$ is the steady state probabilities given by $\left[\Pi(0,0,0), \ldots, \Pi\left(n_{1}, n_{2}, n_{3}\right), \ldots\right]$ and $\mathbf{P}$ is the transition probabilities matrix composed by the valid transitions in the system described in Table I. Now, the average number of packets lost per frame is calculated as:

$$
E\left\{L_{p}(\mathbf{n})\right\}=\sum_{m_{1}=0}^{M} \ldots \sum_{m_{w}=0}^{M-\sum_{i=1}^{w-1} m_{i}} \sum_{k_{1}=0}^{\lceil S\rceil} \ldots \sum_{k_{z}=0}^{\lceil S\rceil-\sum_{i=1}^{z-1} k_{i}} E\left\{L_{p}(\mathbf{n} \mid(\mathbf{m}, \mathbf{k}))\right\} P(\mathbf{m}, \mathbf{k})
$$

The average number of packets generated per frame depends only on the number of active nodes at the beginning of the time slot plus the packets already in the system waiting to be transmitted in the previous frame, that is:

$$
E\left\{\sum_{i=1}^{z} n_{i}\right\}=\sum_{m_{1}=0}^{M} \ldots \sum_{m_{w}=0}^{M-\sum_{i=1}^{w-1} m_{i}} \sum_{k_{1}=0}^{\lceil S\rceil} \ldots \sum_{k_{z}=0}^{\lceil S\rceil-\sum_{i=1}^{z-1} k_{i}} E\left\{\sum_{i=1}^{z} n_{i} \mid(\mathbf{m}, \mathbf{k})\right\} P(\mathbf{m}, \mathbf{k})
$$

Packet dropping probability, $P_{d}$, is found by considering all the possible values of $m_{l}, \ldots, m_{w}, k_{l}, \ldots, k_{z}$, That is:

$$
P_{d}=E\left\{L_{p}(\mathbf{n})\right\} / E\left\{\sum_{i=1}^{z} n_{i}\right\}
$$

\section{NUMERICAL RESULTS}

The goal of this section is to investigate the impact of the primary CHT distribution on the packet level performance of a buffered CRN with VoIP traffic and AMC. A second order (i.e., $w=2$ ) Coxian and log-normal probability distributions for modeling the primary CHT are considered. From the non Markovian distributions used in the literature to model the primary CHT, the log-normal distribution is studied here due to the fact that it is the one that best fits measured data [26]. It is important to remark that, for a fair comparison, the first three standardized moments of the Coxian distribution are respectively matched to the corresponding ones of the lognormal distributions used in this section. For reference, also numerical results are presented for exponentially distributed primary CHT. Numerical results are shown for different values of the first three standardized moments (i.e., mean value, coefficient of variation, and skewness) of the primary CHT distribution and for a target VoIP connection blocking probability of $5 \%$. System performance is evaluated in terms of packet dropping probability and mean packet delay of cognitive users. Unless otherwise specified, the following values of the system parameters were used in the plots of this section: a voice activity factor equals 0.5 (i.e., $P_{O N-O F F}=P_{O F F}$ $\left.{ }_{O N}=2 / 180\right) ; T_{f}=20 \mathrm{~ms}$; total number of channels $M=4$; packet size $b$; transmission rates $R_{b}^{1}$ and $R_{b}^{2}$ have been set to obtain a maximum capacity $C_{\max }=8$ packets per frame. Parameters of the Coxian distributed primary CHT are presented in Table II. In this section, a moderate primary traffic load is considered.
Specifically, a blocking probability for the PUs equals 0.5 is employed. In this section the label "SK" stands for skewness, "CV" stands for coefficient of variation. Also, "EXP-NEG," "Log-Norm," and "COX-2" stand for exponential, log-normal, and $2^{\text {nd }}$ order Coxian distributions, respectively.

TABLE II. PARAMETERS OF THE COXIAN ORDER 2 DISTRIBUTIONS TO ACHIEVE THREE DIFFERENT MEAN VALUES OF THE PRIMARY CHT.

\begin{tabular}{|c|c|c|c|c|c|}
\hline$C V$ & $S K$ & $\gamma$ & $\mu_{1}^{(P)}$ & $\mu_{2}^{(P)}$ & $E\left\{X^{(p)}\right\}$ \\
\cline { 1 - 5 } 3 & 9 & 0.9506 & 0.010482 & $5.8408 \mathrm{e}-4$ & \multirow{2}{*}{$180 \mathrm{~s}$} \\
\cline { 1 - 5 } 9 & 15 & 0.9802 & $5.5448 \mathrm{e}-2$ & $1.2222 \mathrm{e}-4$ & \\
\hline 3 & 9 & 0.9506 & $5.2406 \mathrm{e}-2$ & $2.9204 \mathrm{e}-3$ & \multirow{2}{*}{$36 \mathrm{~s}$} \\
\cline { 1 - 5 } 9 & 15 & 0.9802 & 0.2772 & $6.1112 \mathrm{e}-4$ & \\
\hline 3 & 9 & 0.9506 & $2.0962 \mathrm{e}-3$ & $1.1682 \mathrm{e}-4$ & \multirow{2}{*}{900} \\
\cline { 1 - 5 } 9 & 15 & 0.9802 & $1.109 \mathrm{e}-2$ & $2.4445 \mathrm{e}-5$ & \\
\hline
\end{tabular}

Figs. 2 and 3 show simulation results for the packet dropping probability for different values of the first three standardized moments of the primary CHT distribution. In the plots of Figs. 2 and 3 a 3D representation is used in order to simultaneously visualize the effect of the buffer size and secondary traffic load on the packet dropping probability. In Fig. 2 the cases when the CHT is exponentially and Coxian distributed are presented, while in Fig. 3 the log-normal case is presented. In Figs. 2.a and 3.a, 2.b and 3.b, and 2.c and 3.c, the mean value of the CHT is equal to $180 \mathrm{~s}, 36 \mathrm{~s}$ and $900 \mathrm{~s}$, respectively. Additionally, in Fig. 2 analytical results are also presented. From Fig. 2 perfect agreement between simulation and analytical results is observed which validates our mathematical model developed in Section III. On the other hand, Fig. 4 presents simulation results for the mean packet delay as function of secondary traffic load when the mean primary CHT equals $180 \mathrm{~s}$, for buffer size $Q=10$ packets.

As expected, Figs. 2-4 show that both secondary packet dropping probability and mean packet delay are monotonically increasing function of secondary traffic load. Figs. 2 and 3 show that, for the primary traffic load considered and for a given secondary traffic load, the packet dropping probability remains unchanged as buffer size goes from 10 packets to 25 packets. However, the most relevant result that can be extracted from Figs. 2-4 is the fact that packet dropping probability and mean packet delay are practically insensitive to the distribution used to model the primary CHT. This indicates that, under moderate primary traffic load, the white spaces are equally exploited by the cognitive radio network irrespective of the distribution-type of the primary CHT. Thus, only the secondary traffic load is relevant for the performance (in terms of packet delay and dropping rate) of buffered CRNs with VoIP traffic and AMC. The reason of this behavior is due to the fact that the CRN takes advantage of the available white spaces as soon as these white spaces are available independent of the identity of the particular primary resources employed. Then, for the analytical performance evaluation of a VoIP traffic-based CRN under these system conditions and for the packet delay and dropping rate metrics, the negative exponential distribution is preferred due to its simplicity. 


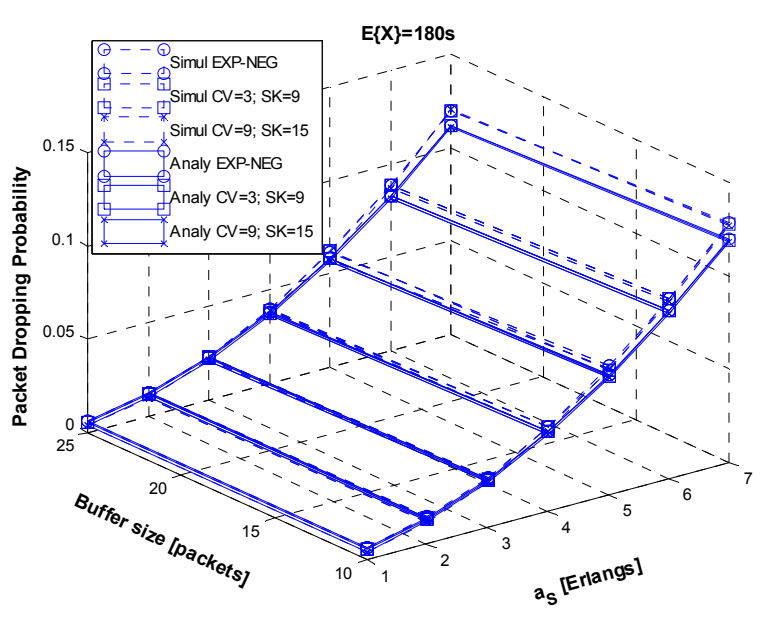

(a)

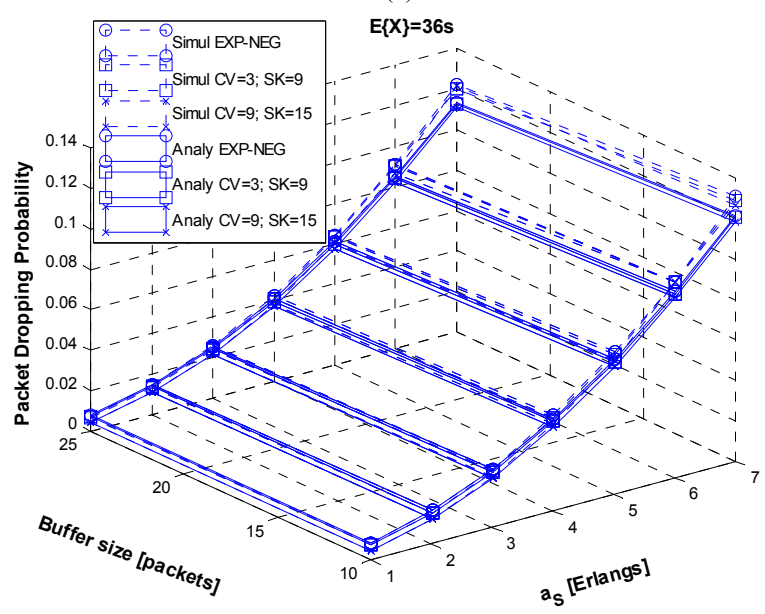

(b)

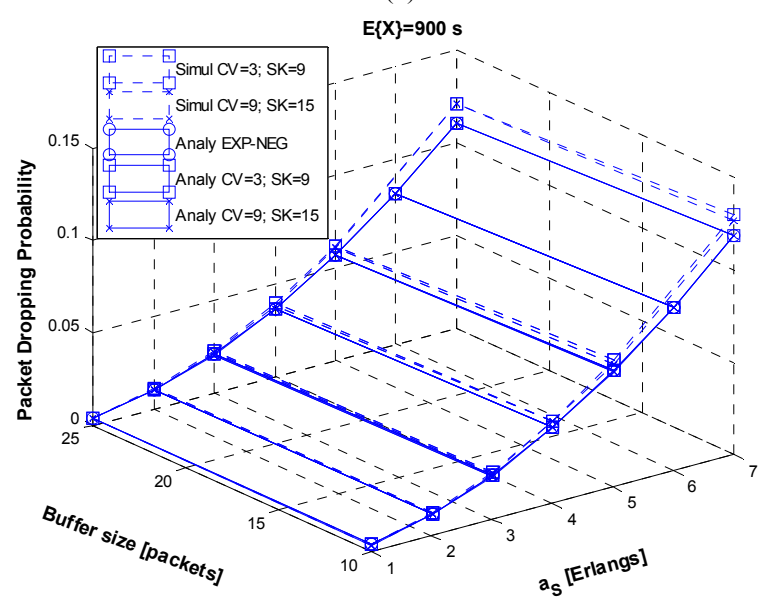

(c)

Fig. 2. Packet dropping probability versus queue size and secondary traffic load for $2^{\text {nd }}$ Coxian and exponentially distributed primary CHT. The mean value of the primary CHT is equal to a) $180 \mathrm{~s}$, b) $36 \mathrm{~s}$, and c) $900 \mathrm{~s}$.

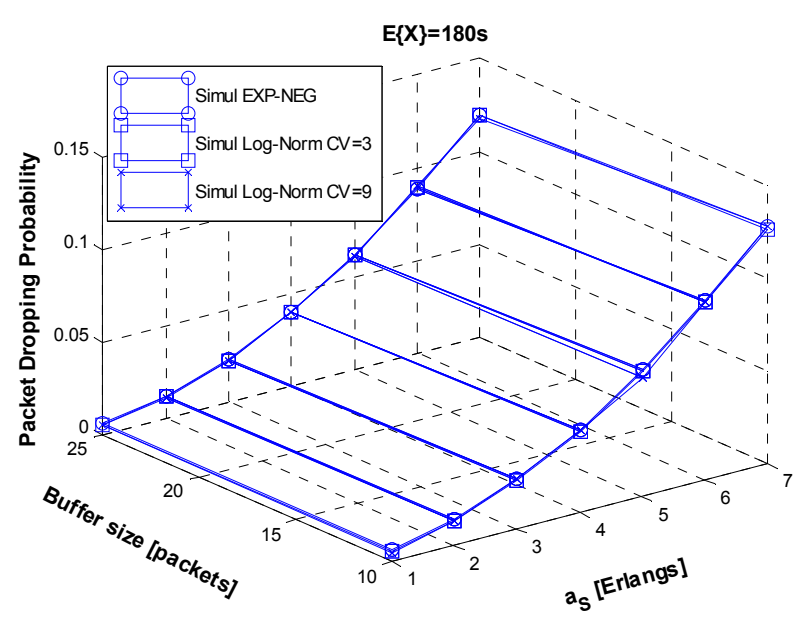

(a)

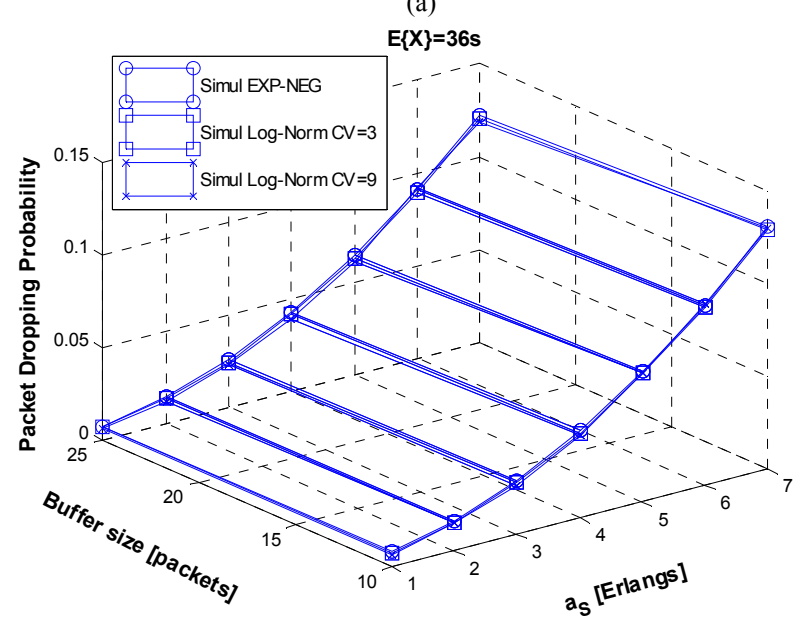

(b)

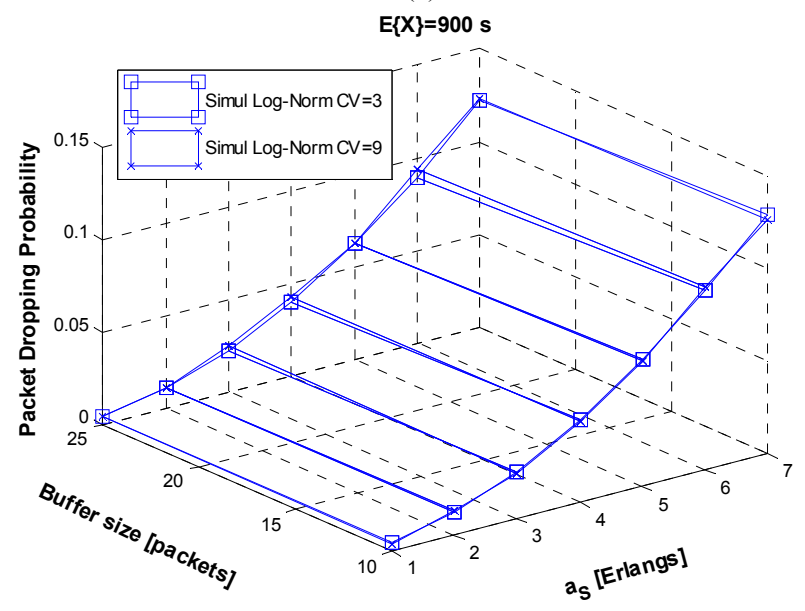

(c)

Fig. 3. Packet dropping probability versus buffer size and secondary traffic load for log-normally and exponentially distributed primary CHT. The mean value of the primary CHT is equal to a) $180 \mathrm{~s}$, b) $36 \mathrm{~s}$, and c) $900 \mathrm{~s}$. 


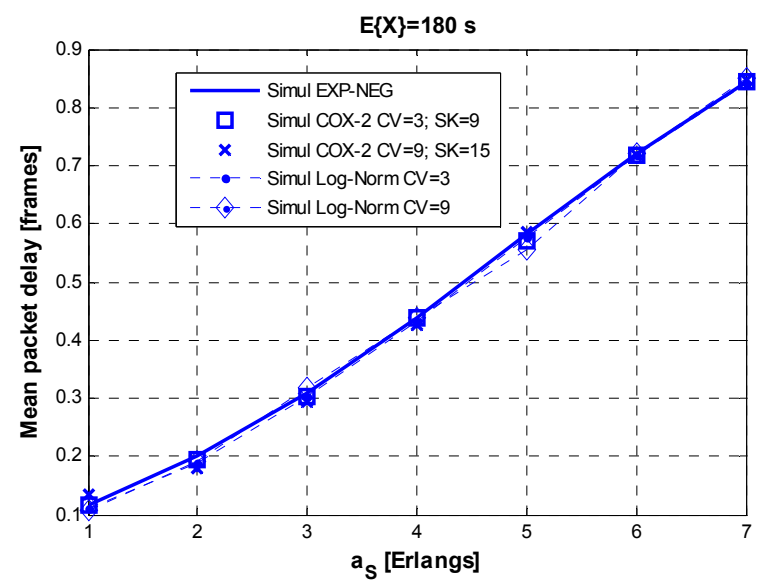

Fig. 4. Mean packet delay as function of secondary traffic load. The mean value of the CHT is equal to 180 s.

\section{CONCLUSIONS}

System performance at the packet level of buffered cognitive radio networks (CRNs) with AMC under VoIP traffic based on different prescribed primary channel holding time (CHT) distribution was analyzed and evaluated. Negative exponential, log-normal, and Coxian distributions were considered to model the primary CHT. From the mathematical point of view, a joint connection and packet level teletraffic analysis considering the primary channel holding time to be Coxian distributed was developed. The novelty of our teletaffic model is the adoption of Coxian distribution instead of the exponential one to match real environment primary CHT distributions. Building on this, the performance of a VoIP traffic-based CRN was evaluated in terms of packet dropping probability and packet delay of cognitive users. Numerical results reveal that for these particular packet level metrics and secondary traffic type, the performance of the CRN is not dependent on the probability distribution of the primary channel holding time but the traffic load of the secondary network. Then, for the analytical performance evaluation of VoIP-traffic based CRNs with AMC and buffering, the negative exponential distribution for modeling primary CHT can be employed with a negligible accuracy penalty on the numerical results.

\section{REFERENCES}

[1] L. Jiang, T. Jiang, Z. Wang, and X. He, "Capacity analysis of VoIP service in cognitive radio system," in Proc. WiCOM'2010, pp. 1-3.

[2] H. Lee and D.-H. Cho, "VoIP capacity analysis in cognitive radio systems," IEEE Commun. Letters, vol. 13, no. 6, June 2009.

[3] H. Lee and D.-H. Cho, "Capacity improvement and analysis of VoIP service in a cognitive radio system," IEEE Trans. Veh. Technol., vol. 59, no. 4, pp. 1646-1651, May 2010.

[4] S. Gunawardeba and W. Zhuang, "Voice capacity of cognitive radio networks," in Proc. IEEE ICC 2010, Cape Town, South Africa, May 2010.

[5] P. Wang, D. Niyato, and H. Jiang "Voice-service capacity analysis for cognitive radio networks," IEEE Trans. Veh. Technol., vol. 59, no. 4, pp. 1779-1790, May 2010.

[6] X. Zhu, L. Shen, and T.-S. P. Yum, "Analysis of cognitive radio spectrum access with optimal channel reservation," IEEE Commun. Lett., vol. 11 , no. 4, pp. 1-3, Apr. 2007.
[7] Y. Zhang, "Dynamic spectrum access in cognitive radio wireless networks," in Proc. IEEE ICC'08, Beijing, China, May 2008, pp. 4927 4932.

[8] W. Ahmed, J. Gao, and M. Faulkner, "Performance evaluation of a cognitive radio network with exponential and truncated usage models," in Proc. IEEE ISWPC'09, Melbourne, Australia, Feb. 2009.

[9] C. Jedrzycki and V. Leung, "Probability distributions of channel holding time in cellular telephony systems," in Proc. VTC'96, May 1996, pp. 247-251.

[10] F. Barceló and J. Jordán, "Channel holding time distribution in public telephony systems (PAMR and PCS)," IEEE Transactions on Vehicular Technology, vol. 49, no. 5, pp. 1615-1625, Sep. 2000.

[11] A. Pattavina and A. Parini, "Modelling voice call interarrival and holding time distributions in mobile network," in Proceedings of the 19th International Teletraffic Congress (ITC-19), 2005.

[12] W. Chen, H. Hung, and Y. Lin, "Modeling VoIP call holding times for telecommunications," IEEE Network, vol. 21, no. 6, pp. 22-28, 2007.

[13] E. Yavuz and V. Leung, "Modeling channel occupancy times for voice traffic in cellular networks," in Proc. ICC '07, 2007, pp. 332-337.

[14] D. Willkomm, S. Machiraju, J. Bolot, and A. Wolisz, "Primary user behavior in cellular networks and implications for dynamic spectrum access," IEEE Communications Magazine, vol. 47, no. 3, pp. 88-95, Mar. 2009

[15] V. Pla, J.-R. Vidal, J. Martinez-Bauset, and L. Guijarro, "Modeling and characterization of spectrum white spaces for underlay cognitive radio networks," in Proc. IEEE ICC'2010, Cape Town, South Africa, 23-27 May 2010.

[16] S.L. Castellanos-López, F.A. Cruz-Pérez, M.E. Rivero-Angeles, and G. Hernández-Valdez, "Impact of the primary resource occupancy information on the performance of cognitive radio networks with VoIP traffic,” in Proc. CROWNCOM'2012, Stockholm, Sweden, June 2012.

[17] S. Ghani and M. Schwartz, "A decomposition approximation for the analysis of voice/data integration," IEEE Trans. Commun., vol. 42, no. 7, Jul. 1994, pp. 2441-2452.

[18] D. Mitra, M. I. Reiman, "Robust dynamic admission control for unified cell and call QoS in statistical multiplexers," IEEE Journal on Selected Areas on Communications, vol. 16, no. 5, Jun. 1998, pp. 692-707.

[19] S.L. Castellanos-López, F.A. Cruz-Pérez, M.E. Rivero-Ángeles, and G. Hernández-Valdez, "Joint connection level and packet level analysis of cognitive radio networks with VoIP traffic," IEEE Journal on Selected Areas in Communications -Cognitive Radio Series, vol. 32, no. 3, pp. 601-614, March, 2014.

[20] P.T. Brady, "A model for generating ON-OFF speech patterns in twoway conversations," Bell Syst. Technol. J., vol. 48, no. 7, pp. 2445-2472, Sept. 1969.

[21] Q. Bi, S. Vitebsky, Y. Yang, Y. Yuan, and Q. Zhang, "Performance and capacity of cellular OFDMA systems with voice-over-IP traffic," IEEE Trans. Veh. Technol., vol. 57, no. 6, pp. 3641-3652, Nov. 2008.

[22] S. Karapantazis, F.-N- Pavlidou, "VoIP: A comprehensive survey on a promising technology," Comp. Networks, vol. 53, pp. 2050-2090, 2009.

[23] S. Garg and M. Kappes, "Can I add a VoIP call?," in Proc. IEEE ICC'2003, Anchorage, AK, May 2003, pp. 779-783.

[24] S.L. Castellanos-López, F.A. Cruz-Pérez, and G. Hernández-Valdez, "Performance evaluation of cognitive radio systems with Coxian distributed channel holding time in the primary network," in Proc. IEEE WCNC'2011, Cancun, Quintana-Roo, Mexico, pp. 1218-1223, Mar. 2011.

[25] B. Sinclair, "Coxian Distributions," Connexions module: m10854. Available on line: http://cnx.org/content/m10854/latest/

[26] C. Schirmer, M.A. Alsharef, W. Kotterman, A. Ihlow, G. Del Galdo, A Heuberger, "High time-resolution spectrum occupancy model for testing of cognitive radio devices," in Proc. $24^{\text {th }}$ IEEE PIMRC'2013, London, UK, Sep. 2013, pp. 596-600.

[27] M. Lopez-Benitez and F. Casadevall, "Empirical time-dimension model of spectrum use based on a discrete-time markov chain with deterministic and stochastic duty cycle models," IEEE Trans. Veh. Technol., vol. 60, no. 6 , pp. 2519-2533, 2011 . 